\title{
Infinite-Dilution Viscoelastic Properties of Polystyrene in $\theta$-Solvents and Good Solvents
}

\author{
Robert M. Johnson, John L. Schrag, and John D. Ferry \\ Department of Chemistry and Rheology Research Center, University of Wisconsin, \\ Madison, Wisconsin 53706, U.S.A.
}

(Received October 3, 1970)

\begin{abstract}
The Birnboim-Schrag multiple-lumped resonator has been used to measure storage $\left(G^{\prime}\right)$ and loss $\left(G^{\prime \prime}\right)$ shear moduli, in the concentration range $(c)$ from 0.0015 to $0.018 \mathrm{~g} / \mathrm{cc}$, of a polystyrene of molecular weight 860000 in two $\theta$-solvents (decalin and di-2-ethylhexyl phthalate) and two good solvents ( $\alpha$-chloronaphthalene and Aroclor 1232), as well as a polystyrene of molecular weight 410000 in Aroclor 1232. The frequency range was from 106 to $6060 \mathrm{~Hz}$. Comparison of the initial concentration dependences of $G^{\prime}$ and $G^{\prime \prime}$ (i.e., the initial slopes of plots of $G^{\prime} / c$ and $\left(G^{\prime \prime}-\omega \eta_{s} / c\right.$, where $\omega$ is radian frequency and $\eta_{s}$ solvent viscosity), and examination of their changes with frequency, show that the longest relaxation time is affected much more than others by incipient intermolecular interactions. The intercepts, $\left[G^{\prime}\right]$ and $\left[G^{\prime \prime}\right]$, of these extrapolation plots and their frequency dependence can be compared with infinitedilution theories. In the two $\theta$-solvents, they agree closely with the Zimm theory; the proportionality of relaxation times to $\eta_{s}$ and the 23 -fold difference in $\eta_{s}$ affords an extended scale of reduced frequency. In the two good solvents, they fit the Tschoegl theory with hydrodynamic parameter $h=40$ and the non-Gaussian parameter $\varepsilon=0.13$.

KEY WORDS Viscoelasticity / Dilute Solutions / Multiple-lumped Resonator / Concentration Dependence / Intrinsic Modulus / Zimm Theory / Polystyrene /
\end{abstract}

Extensive measurements of dynamic viscoelastic properties of several polymers have been made previously ${ }^{1}$ at concentrations of the order of $0.01 \mathrm{~g} / \mathrm{cc}$, in high viscosity solvents over a continuous frequency range from 0.01 to 400 $\mathrm{Hz}$, and significant conclusions have been drawn concerning the applicability of various molecular theories. ${ }^{2}$ However, even at this low concentration, intermolecular interactions prevent quantitative comparison with current theories; it is necessary to extrapolate data to infinite dilution. Successful extrapolation was accomplished by Tanaka and Sakanishi ${ }^{3}$ by use of piezoelectric torsional crystals ${ }^{4}$ with three frequencies in the range from 20 to $120 \mathrm{kHz}$, the solvent viscosities being of the order of a centipoise. They concluded that at infinite dilution the behavior in $\theta$-solvents corresponded to the theory of Zimm, ${ }^{5}$ whereas in good solvents it could be described by the theory of Tschoegl ${ }^{6}$ with intermediate hydrodynamic interaction.

A new experimental method, ${ }^{7}$ following a principle originally suggested by Birnboim, ${ }^{8}$ permits measurements at a larger number of discrete frequencies in the range from 100 to $8300 \mathrm{~Hz}$, at polymer concentrations as low as $0.0015 \mathrm{~g} / \mathrm{cc}$. The appropriate solvent viscosity range is of the order of 0.005 to 0.5 poise. In the present paper, data are reported for a high-molecular weight polystyrene in two theta solvents and two good solvents, as well as another polystyrene of lower molecular weight in one good solvent. The nature of the inital concentration dependence of the storage and loss shear moduli $G^{\prime}$ and $G^{\prime \prime}$ is examined, and the results of extrapolation to infinite dilution are compared with molecular theories.

\section{THEORY OF INITIAL CONCENTRATION DEPENDENCE}

Although the primary interest of this investigation is in the extrapolated data corresponding to infinite dilution conditions, it is desirable 
to examine the extrapolation process and extract additional information from the concentration dependence of measured quantities at very low concentrations if possible. Some features of the initial concentration dependence of $G^{\prime}$ and $G^{\prime \prime}$ can be inferred from the well-known dependence of steady flow viscosity, $\eta$ :

$$
\eta-\eta_{s}=[\eta] \eta_{s} c+k^{\prime} \eta_{s}[\eta]^{2} c^{2}+0\left(c^{3}\right)
$$

where $[\eta]$ is the intrinsic viscosity, $\eta_{s}$ solvent viscosity, $c$ concentration, and $k^{\prime}$ the Huggins constant. In most molecular theories of linear viscoelasticity, $\eta-\eta_{s}$ can be expressed as

$$
\eta-\eta_{s}=\Sigma G_{p} \tau_{p}
$$

where $\tau_{p}$ is a relaxation time and $G_{p}$ is a "relaxation strength"; in the theories of Rouse, Zimm, and Tschoegl, all $G_{p}$ are equal to $c R T / M$, where $M$ is the (uniform) molecular weight, $R$ is the gas constant, and $T$ is absolute temperature.

As $c \rightarrow 0, G_{p}$ is directly proportional to $c$ and the $\tau_{p}$ approach constant values corresponding to isolated molecules with no interactions; this corresponds to the linear term in eq 1 . We shall assume that the square term arises from a linear dependence of $\tau_{p}$ on $c$ while the $G_{p}$ remain directly proportional to $c$. Two limiting cases may be distinguished: (i) all $\tau_{p}$ depend linearly on $c$ in the same manner, viz.,

$$
\tau_{p}=\tau_{0 p}(1+a c)
$$

or (ii) only the longest relaxation time is affected by concentration as the polymer molecules just begin to interact with increasing concentration, viz.,

$$
\tau_{1}=\tau_{01}\left(1+a_{1} c\right), \text { all other } \tau_{p}=\tau_{0 p}
$$

Case (ii) appears to be more probable a priori.

\section{All Relaxation Times Concentration-Dependent}

In case (i), substitution of eq 3 into eq 2 and comparison with eq 1 identifies $a=k^{\prime}[\eta]$. The concentration dependences of $G^{\prime}$ and $G^{\prime \prime}$ can be rather simply expressed for the limited cases of the Rouse and Zimm theories at low frequencies and at higher frequencies where $G^{\prime}$ and $G^{\prime \prime}$ are proportional to a fractional power of $\omega$. It is convenient to specify the ratio $\gamma^{\prime}$ between slope and intercept when $G^{\prime} / c$ is plotted against $c$; the corresponding ratio $\gamma^{\prime \prime}$ when
$\left.G^{\prime \prime}-\omega \eta_{s}\right) / c$ is plotted against $c$; and the dimensionless ratio $\gamma=\gamma^{\prime} / \gamma^{\prime \prime}$.

At low frequencies, where all $\omega \tau_{p} \ll 1$,

$$
\begin{gathered}
G^{\prime}=(c R T / M) \sum_{p} \omega^{2} \tau_{p}^{2} /\left(1+\omega^{2} \tau_{p}^{2}\right) \\
\cong(c R T / M) \sum \omega^{2} \tau_{0 p}^{2}(1+a c)^{2} \\
\gamma^{\prime}=\lim _{c \rightarrow 0}\left(G^{\prime} / c\right)^{-1} d\left(G^{\prime} / c\right) / d c=2 a \\
G^{\prime \prime}-\omega \eta_{s}=(c R T / M) \sum_{p} \omega \tau_{p} /\left(1+\omega^{2} \tau_{p}^{2}\right) \\
\cong(c R T / M) \sum \omega \tau_{0 p}(1+a c) \\
\gamma^{\prime \prime}=a \\
\gamma=\gamma^{\prime} / \gamma^{\prime \prime}=2
\end{gathered}
$$

Here $\omega$ is the radian frequency.

In the power law region of frequencies, the results depend on whether the Rouse or the Zimm theory is followed. For Rouse, ${ }^{9}$

$$
\begin{aligned}
G^{\prime} & =G^{\prime \prime}-\omega \eta_{s} \\
& =(c R T / M)(\pi / 2 \sqrt{2}) \omega^{1 / 2} \tau_{0 p}{ }^{1 / 2}(1+a c)^{1 / 2}
\end{aligned}
$$

whence

$$
r^{\prime}=\gamma^{\prime \prime}=a / 2
$$

and for Zimm, ${ }^{9} G^{\prime}$ and $G^{\prime \prime}-\omega \eta_{s}$ are both proportional to $\omega^{2 / 3} \tau_{0 p}{ }^{2 / 3}(1+a c)^{2 / 3}$, leading to

$$
\gamma^{\prime}=\gamma^{\prime \prime}=2 a / 3
$$

Thus in the neighborhood of $\omega=\tau_{1}^{-1}$ we expect $\gamma^{\prime}$ and $\gamma^{\prime \prime}$ to decrease monotonically with increasing frequency-and hence extrapolation of data to infinite dilution will become easier.

\section{Only Longest Relaxation Time Concentration-} Dependent

In case (ii), substitution of eq 4 into eq 2 and comparison with eq 1 identifies $a_{1}=S k^{\prime}[\eta]$, where $S=\sum_{p}\left(\tau_{p} / \tau_{1}\right)$, a rapidly converging sum which is 1.645 for the Rouse theory and 2.369 for the Zimm theory. At low frequencies, the analogs of eq 5 to 9 are

$$
\begin{gathered}
G^{\prime}=(c R T / M)\left[\sum_{p} \omega^{2} \tau_{0 p}{ }^{2}+\omega^{2} \tau_{01}{ }^{2}\left(2 a_{1} c+a^{\beth^{2}} c^{2}\right)\right] \\
\gamma^{\prime}=2 a_{1} / S^{\prime} \\
G^{\prime \prime}-\omega \eta_{s}=(c R T / M)\left(\sum_{p} \omega \tau_{0 p}+\omega \tau_{01} a_{1} c\right) \\
\gamma^{\prime \prime}=a_{1} / S \\
\gamma=2 S / S^{\prime} ; \quad 3.04 \text { for Rouse, } 4.10 \text { for Zimm }
\end{gathered}
$$


Here $S^{\prime}=\sum_{p}\left(\tau_{p} / \tau_{1}\right)^{2}$, which is 1.082 for Rouse and 1.156 for Zimm.

In the power-law region of frequencies, $\gamma^{\prime}$ and $\gamma^{\prime \prime}$ are essentially zero because only the first term in the series has concentration dependence and the other terms make the major contribution to both $G^{\prime}$ and $G^{\prime \prime}$. Thus extrapolation will become very easy at higher frequencies.

It may be noted that, according to the form of eq 5 and 13, plots of $G^{\prime} / c$ against $c$ are expected to be parabolic rather than linear (until higher order terms of concentration dependence of $\tau$ 's come in) and a plot of $\left(G^{\prime} / c\right)^{1 / 2}$ against $c$ should be linear over a larger concentration range.

The features of these predictions are summarized in Table I. Here the subscript PL

Table I. Features of initial concentration dependence based on concentration dependence of relaxation times

\begin{tabular}{|c|c|c|c|c|}
\hline & \multicolumn{2}{|c|}{ Case (i) } & \multicolumn{2}{|c|}{ Case (ii) } \\
\hline & \multicolumn{2}{|c|}{ All $\tau$ affected } & \multicolumn{2}{|c|}{ Only $\tau_{1}$ affected } \\
\hline & Rouse & Zimm & Rouse & Zimm \\
\hline$\gamma_{L}$ & 2 & 2 & 3.04 & 4.10 \\
\hline$\gamma^{\prime} P L / \gamma^{\prime}{ }_{L}$ & $1 / 4$ & $1 / 3$ & 0 & 0 \\
\hline$\gamma^{\prime \prime}{ }_{P L} / \gamma^{\prime \prime}{ }_{L}$ & $1 / 2$ & $2 / 3$ & 0 & 0 \\
\hline
\end{tabular}

refers to the power-law region of frequencies, and $L$ refers to low frequencies. Evidently, cases (i) and (ii) should be clearly distinguishable from experimental data, and the Rouse and Zimm theories also give perceptibly different predictions. Of course, case (ii) is an oversimplification; the longest relaxation time may be the first to be affected by increasing concentration, but others are no doubt successively affected though to lesser degrees.

An analysis of the modes of motion in the bead-spring model taking into account pairwise interactions of molecules has been made by Ogasa and Imai. ${ }^{10}$ It predicts that in $\theta$-solvents (corresponding to the Zimm theory) $\gamma^{\prime}$ and $\gamma^{\prime \prime}$ should be proportional to $\omega^{-1 / 3}$.

\section{EXPERIMENTAL}

\section{Materials}

The two polystyrenes were synthesized by the Pressure Chemical Company. Sample 6a had a weight-average molecular $\left(M_{w}\right)$ of 860000 and weight to number average ratio $<1.15$; it was reprecipitated from a $1 \%$ solution in benzene by methanol (perhaps improving the sharpness of molecular weight distribution somewhat) and dried in vacuo. Sample $3 \mathrm{a}$ had $M_{w}=410000$ and $M_{w} / M_{n}<1.10$. It was dried in vacuo without further purification.

The solvent had the following characteristics. Decalin was obtained from J. T. Baker Chemical Company. The proportion of cis isomer was about $54 \%$ as determined from both density measurement and gas chromatogram. At the $\theta$ temperature for polystyrene, ${ }^{11} 16^{\circ} \mathrm{C}$, the viscosity was 0.0295 poise and the density $0.8868 \mathrm{~g} / \mathrm{cc}$. Di-2-ethylhexyl phthalate (DOP) was obtained from Union Carbide Chemicals Corporation. At the $\theta$-temperature for polystyrene, $22^{\circ} \mathrm{C}$, the viscosity was 0.678 poise and the density $0.9827 \mathrm{~g} /$ cc. $^{12} \alpha$-Chloronaphthalene $(\alpha-\mathrm{C} 1 \mathrm{~N})$ was obtained from Matheson, Coleman, and Bell Chemical Company, boiling range 133.0 to $135.0^{\circ} \mathrm{C}$. The viscosity at $25.0^{\circ} \mathrm{C}$ was 0.0318 poise, and the density $1.195 \mathrm{~g} / \mathrm{cc}$. Aroclor 1232, lot 9/66, was obtained from Monsanto Chemical Company. Its viscosity at $25.0^{\circ} \mathrm{C}$ was 0.142 poise and the density $1.269 \mathrm{~g} / \mathrm{cc}$.

Solutions were prepared by weight, and their densities and concentrations in $\mathrm{g} / \mathrm{cc}$ were calculated assuming additivity of volumes. Mixing was accomplished by gentle swirling or with a magnetic stirrer at moderate speeds, at room temperature. The most concentrated solution (about $0.018 \mathrm{~g} / \mathrm{cc}$ for $\theta$-solvents, 0.01 $\mathrm{g} / \mathrm{cc}$ for good solvents) was usually prepared first and diluted serially after each measurement, to conserve polymer, since about $43 \mathrm{cc}$ are required for measurement with the multiplelumped resonator apparatus.

\section{Method}

The multiple-lumped resonator method and the data acquisition and processing system used for these measurements have been described elsewhere. ${ }^{7,13}$ With resonators IIIA and IVA, nine discrete frequencies from 106 to $6060 \mathrm{~Hz}$ were provided. The polystyrene with molecular weight 860000 was measured in both $\theta$-solvents at their respective $\theta$-temperatures; its intrinsic 
Infinite-Dilution Viscoelastic Properties
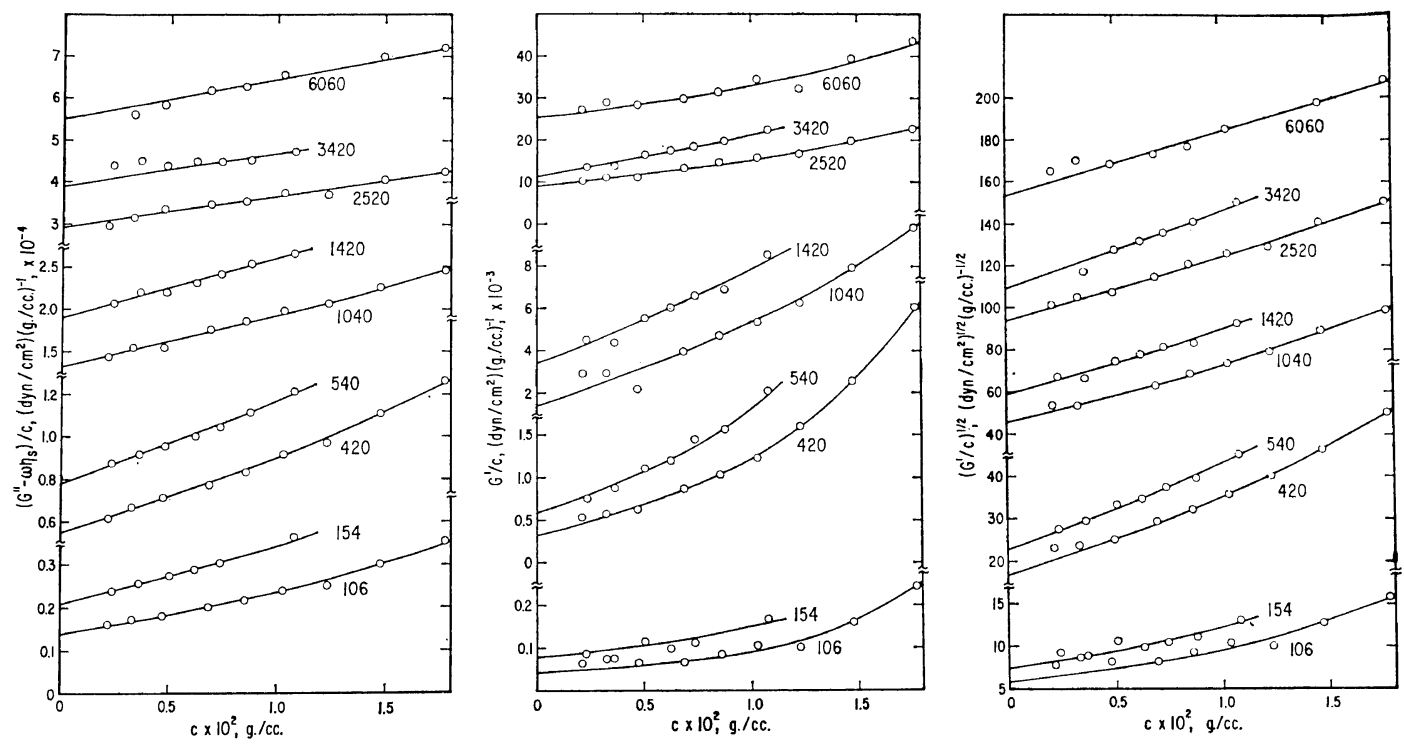

Figure 1. Plots of $\left(G^{\prime \prime}-\omega \eta_{s}\right), c, G^{\prime} / c$, and $\left(G^{\prime} / c\right)^{1 / 2}$ against $c$, each at 9 frequencies as indicated, for $M=860000$ in Decalin. Note that several different ordinate scales are used in each panel.

viscosity was $76 \mathrm{cc} / \mathrm{g}$ in decalin as derived from these measurements. (No independent measurement of $[\eta]$ in DOP was made, but the value in decalin agrees exactly with that calculated for cyclohexane $\mathrm{e}^{14}$ and is presumably applicable in DOP as well.) This polymer was also measured in both good solvents at $25^{\circ} \mathrm{C}$; [ $\left.\eta\right]$ was $197 \mathrm{cc} / \mathrm{g}$ in $\alpha-\mathrm{C} 1 \mathrm{~N}$ and $183 \mathrm{cc} / \mathrm{g}$ in Aroclor 1232 , so the thermodynamic interactions in the two were quite similar, the primary difference being in the solvent viscosity. The polystyrene with molecular weight 410000 was measured in Aroclor 1232 only; $\eta]$ was $111 \mathrm{cc} / \mathrm{g}$.

\section{RESULTS AND DISCUSSION}

\section{Concentration Dependence and Extrapolation}

Most of the data are given here in graphical form; numerical data are available elsewhere. ${ }^{15}$ In Figure $1,\left(G^{\prime \prime}-\omega \eta_{s}\right) / c$ and $G^{\prime} / c$ are plotted against $c$ for $M=860000$ in the $\theta$-solvent decalin. The curvature is more pronounced for the latter, as expected from the deduction that the latter is parabolic in a concentration range where the former is linear. In the third panel, $\left(G^{\prime} / c\right)^{1 / 2}$ is plotted against $c$ with the expected reduction in curvature; extrapolated values of $\left[G^{\prime}\right]$ $=\lim _{c \rightarrow 0}\left(G^{\prime} / c\right)$ were obtained by squaring the intercepts from this plot. It should be noted that several different vertical scales are employed.

In Figure 2, similar plots are shown for $M$ $=860000$ in the good solvent $\alpha$-chloronaphthalene. The concentration range is somewhat less because of the higher intrinsic viscosity; the measured quantities are larger and concentration dependence is more pronounced. The Huggins constant is lower $(0.40$ compared with 0.76 in decalin) and the curvature is less pronounced in each panel; $\left(G^{\prime \prime}-\omega \eta_{s}\right) / c$ and $\left(G^{\prime} / c\right)^{1 / 2}$ are essentially linear over the range covered. Extrapolations in the other $\theta$-solvent and the other good solvent were similar in character, respectively.

Because of the different vertical scales in Figures 1 and 2, trends in slopes with frequency are not apparent. It is instructive to normalize the slope by the intercept by plotting $\left(G^{\prime \prime}-\omega \eta_{s} / c\right) /\left[G^{\prime \prime}\right]$ and $\left(G^{\prime} / c\right)^{1 / 2} /\left[G^{\prime}\right]^{1 / 2}$ as in Figure 3 , taken from alternate curves in the first and third panels of Figure 2. Here $\left[G^{\prime \prime}\right]$ is $\lim _{c \rightarrow 0}\left(G^{\prime \prime}-\omega \eta_{s}\right) / c$. The slopes in the first panel are values of $\gamma^{\prime \prime}$; those in the second 
R. M. Johnson, J. L. Schrag, and J. D. Ferry
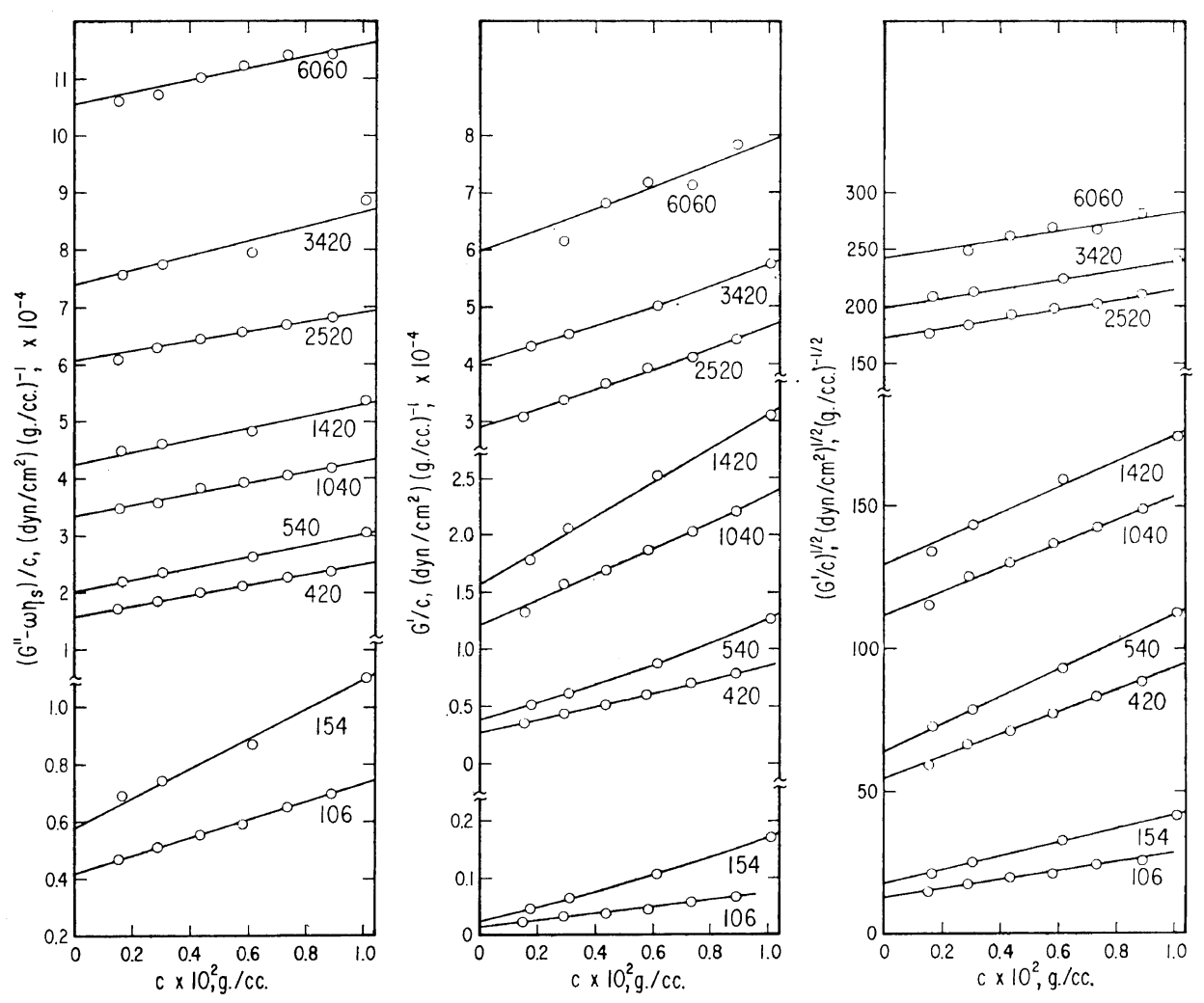

Figure 2. Plots as in Figure 1 for $M=860000$ in $\alpha$-chloronaphthalene.
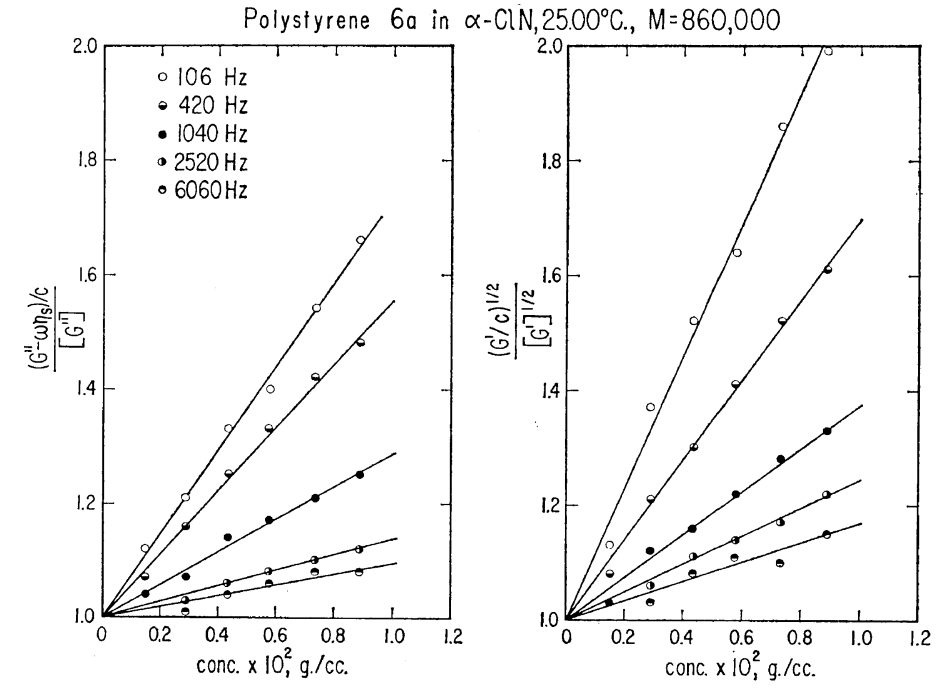

Figure 3. Data from the first and third panels of Figure 2, each line normalized by its intercept, for 5 frequencies as indicated. 
panel are $\gamma^{\prime} / 2$. Evidently, both $\gamma^{\prime}$ and $\gamma^{\prime \prime}$ decrease monotonically and rather rapidly with increasing frequency. Corresponding plots for other solvents were similar in character.

Values of $\gamma^{\prime}, \gamma^{\prime \prime}$, and $\gamma$ for this sample in decalin and $\alpha-\mathrm{C} 1 \mathrm{~N}$ are listed in Table II.

Table II. Parameters describing initial concentration dependence, $M=860000$

\begin{tabular}{|c|c|c|c|c|c|c|}
\hline \multirow{2}{*}{ Frequency } & \multicolumn{3}{|c|}{ In decalin } & \multicolumn{3}{|c|}{$\begin{array}{l}\text { In } \alpha \text {-chloro- } \\
\text { naphthalene }\end{array}$} \\
\hline & $\gamma^{\prime}$ & $\gamma^{\prime \prime}$ & $\gamma$ & $\gamma^{\prime}$ & $\gamma^{\prime \prime}$ & $\gamma$ \\
\hline 106 & 200 & 57 & 3.5 & 246 & 74 & 3.3 \\
\hline 154 & 200 & 57 & 3.5 & 266 & 88 & 3.0 \\
\hline 420 & 222 & 54 & 3.7 & 138 & 56 & 2.5 \\
\hline 540 & 166 & 46 & 3.6 & 150 & 48 & 3.1 \\
\hline 1040 & 104 & 44 & 2.4 & 75 & 28 & 2.6 \\
\hline 1420 & 92 & 37 & 2.5 & 70 & 25 & 2.8 \\
\hline 2520 & 60 & 24 & 2.5 & 48 & 14 & 3.4 \\
\hline 3420 & 62 & 18 & 3.4 & 40 & 17 & 2.4 \\
\hline 6060 & 40 & 17 & 2.3 & 33 & 10 & 3.3 \\
\hline
\end{tabular}

Two aspects are of significance in connection with the calculated values in Table I. In the first place, the drop in $\gamma^{\prime}$ and $\gamma^{\prime \prime}$ with increasing frequency is considerably more pronounced than predicted for case (i); in $\alpha-\mathrm{C} 1 \mathrm{~N}$, in particular, $\gamma^{\prime}$ falls by a factor of 0.13 and $\gamma^{\prime \prime}$ by 0.14. (Actually, the slopes in the first and third panels of Figures 1 and 2 are approximately independent of frequency above $\omega \tau_{1}=1$, so that $\gamma^{\prime}$ and $\gamma^{\prime \prime}$ are inversely proportional to $\omega^{2 / 3}$ in a $\theta$-solvent.) Although the value of zero expected at higher frequencies for case (ii) is not reached, it may be concluded that the longest relaxation time is disproportionately affected at very low concentrations. The same conclusion is reached from the magnitude of $\gamma$ at low frequencies; the values of $3.6 \pm 0.1$ in decalin and something over 3 in $\alpha$-C1N clearly exclude case (i). The value in decalin approaches that of 4.1 expected for case (ii) for the Zimm theory in a $\theta$-solvent; the difference is probably associated with a gradually diminishing effect of concentration on relaxation times with indices greater than 1 . The other data were similar in character.
Polystyrene in $2 \Theta-$ Solvents, $M=860,000$

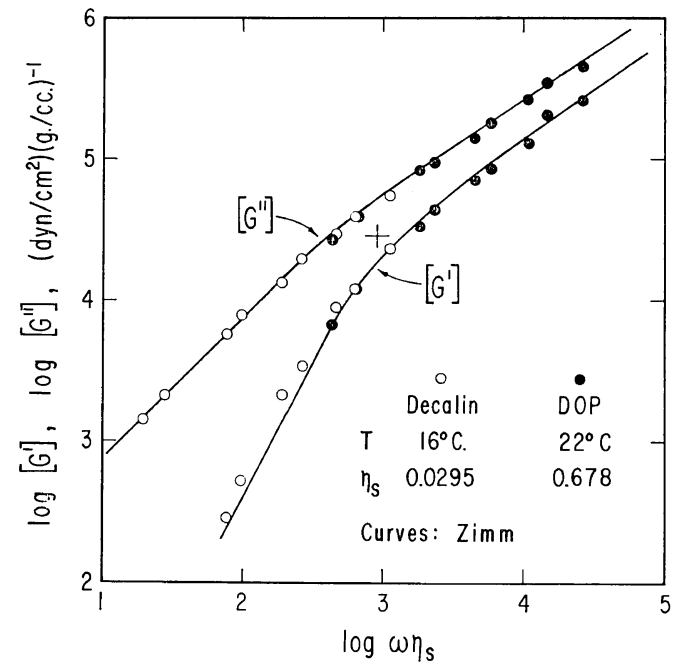

Figure 4. The intrinsic shear moduli $\left[G^{\prime}\right]$ and $\left[G^{\prime \prime}\right]$ plotted logarithmically against $\omega \eta_{s}$ for $M=860000$ in the two $\theta$-solvents: open circles, Decalin; black circles, DOP. Data in DOP shifted by $\log (289 / 295)$ $=-0.01$ on both axes to reduce to $\theta$-temperature of decalin. Curves: Zimm theory. The cross corresponds to $\omega \tau_{01}=1$ and an ordinate of $\log (R T / M)$.

\section{Extrapolated Viscoelastic Functions}

In Figure 4, the intercepts from Figure 1 and similar values of $\left[G^{\prime}\right]$ and $\left[G^{\prime \prime}\right]$ in the other $\theta$-solvent, DOP, are plotted logarithmically against the product $\omega \eta_{s}$, each at its $\theta$-temperature. According to the Rouse-Zimm theories, ${ }^{9}$ $\left[G^{\prime}\right] M / R T$ and $[G]^{\prime \prime} M / R T$ are universal functions of $\omega \tau_{1}=\omega \eta_{s}[\eta] M / R T S$. Hence in combining data for two $\theta$-solvents, in which $[\eta]$ should be the same, use of the abscissa $\omega \eta_{s}$ should result in a single curve for each component of the intrinsic modulus-except for the difference in the two $\theta$-temperatures. To allow for the latter effect, both ordinates and abscissas for the DOP points have been very slightly adjusted by $\log (289 / 295)$ to reduce them to $16^{\circ} \mathrm{C}$, the $\theta$-temperature of decalin. The data in the two solvents combine well to give single curves, confirming the proportionality of relaxation times to $\eta_{s}$. Moreover, the points are in excellent agreement with curves calculated from the theory of Zimm, taking the weight-average molecular weight, with no adjustable parameters. 
Polystyrene in 2 Good Solvents, $M=860,000$

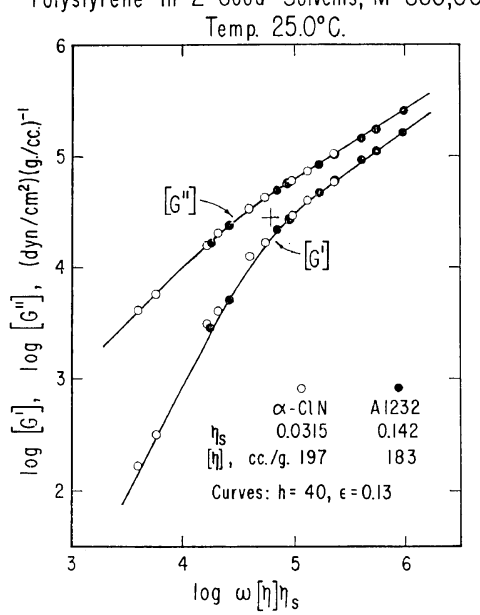

Figure 5. Logarithmic plots of $\left[G^{\prime}\right]$ and $\left[G^{\prime \prime}\right]$ against $\omega \eta_{s}[\eta]$ for $M=860000$ in two good solvents at $25^{\circ} \mathrm{C}$ : open circles, $\alpha$-chloronaphthalene; black circles, Aroclor 1232. Curves: Tschoegl theory with $h=40, \varepsilon=0.13$. Cross as in Figure 4 .

The calculated curves in Figure 4 are based on the original Zimm eigenvalues. ${ }^{5}$ Improved calculations are available from the derivations of Pyun and Fixman ${ }^{16}$ as well as exact matrix solutions by Lodge and $\mathrm{Wu} .{ }^{17}$ However, these will affect the final values only slightly and no attempt has been made to take them into account pending consideration of internal viscosity effects at higher frequencies which will be discussed in a later communication.

It may be noted that the $\left[G^{\prime}\right]$ data at the lowest frequencies fall somewhat above the calculated curves. This would be expected ${ }^{18}$ from a slight distribution in molecular weights.

In Figure 5, similar extrapolated data are plotted from the intercepts of Figure 2 and corresponding values of $\left[G^{\prime}\right]$ and $\left[G^{\prime \prime}\right]$ in the other good solvent, Aroclor 1232 , at $25^{\circ} \mathrm{C}$. Here since the intrinsic viscosities are slightly different it is necessary to use $\omega \eta_{s}[\eta]$ as the abscissa to combine the two sets of data; the $[\eta]$ 's are close enough so that the thermodynamic interactions are essentially the same and no difference in the shape of the frequency dependence would be expected. In fact, data from the two solvents again fall on a single curve for each component of the modulus,

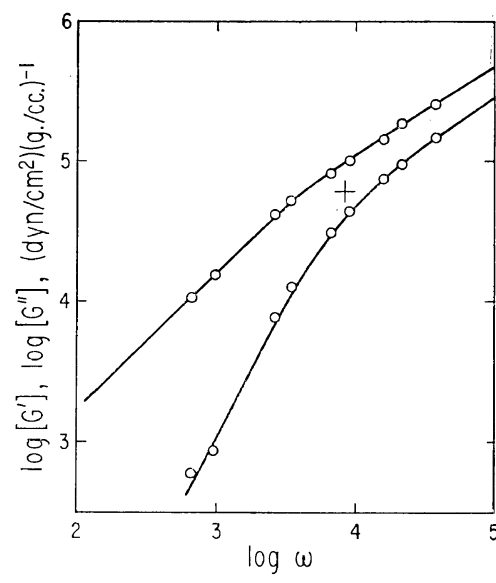

Figure 6. Logarithmic plots of $\left[G^{\prime}\right]$ and $\left[G^{\prime \prime}\right]$ against $\omega$ for $M=410000$ in Aroclor 1232. Curves: Tschoegl theory with $h=40, \varepsilon=0.13$. Cross as in Figure 4.

confirming the proportionality of the $\tau$ 's to $\eta_{s}[\eta]$. However, in the good solvent the Zimm theory does not agree with the data even at infinite dilution. They can be fit quite well with the Tschoegl theory, which involves two parameters, $h$ and $\varepsilon$. The non-Gaussian parameter $\varepsilon$ can be estimated as 0.13 from earlier calculations. ${ }^{1 \mathrm{c}}$ The strength of the hydrodynamic interaction $h(\infty$ in the Zimm theory) is an adjustable parameter; a good fit is achieved with $h=40$.

Similar data on the polystyrene with $M$ $=410000$ in Aroclor 1232, extrapolated to infinite dilution, are plotted logarithmically against $\omega$ in Figure 6 . These data are also fit very well by the Tschoegl theory with $h=40, \varepsilon=0.13$. For these values of the parameters, $S=2.166$ and $\log \tau_{1}=-3.92$.

These results thus confirm the conclusions of Tanaka and Sakanishi, ${ }^{3}$ with more confidence due to the wider frequency range, closer spacing of frequencies, and probably better precision, that at infinite dilution in $\theta$-solvents the viscoelastic behavior of a linear polymer in small oscillations agrees quantitatively with the Zimm theory whereas in good solvents the hydrodynamic interaction appears to be of intermediate character.

The proportionality of relaxation times to solvent viscosity implies that the frictional 
resistance to motions of the macromolecule is provided entirely by the environment and is not influenced by its internal structure, at least ni the frequency range of the present experiments.

Acknowledgment. This work was supported in part by grants from the National Institute of Health and the National Science Foundation. We are indebted to Dr. Dennis J. Massa for supervising the data acquisition and processing system, and to Dr. K. Osaki and Mr. Y. Mitsuda for help with some of the experiments.

\section{REFERENCES}

1. (a) R. B. De Mallie, Jr., M. H. Birnboim, J. E. Frederick, N. W. Tschoeg1, and J. D. Ferry, J. Phys. Chem., 66, 536 (1962); (b) N. W. Tschoegl and J. D. Ferry, ibid., 68, 867 (1964); (c) J. E. Frederick, N. W. Tschoeg1, and J. D. Ferry, ibid., 68, 1974 (1964).

2. J. D. Ferry, "Viscoelastic Properties of Polymers," 2nd ed., Wiley, New York, N.Y. 1970.

3. (a) H. Tanaka, A. Sakanishi, M. Kaneko, and J. Furuichi, J. Polym. Sci., Part C, 15, 317 (1966); (b) A. Sakanishi and H. Tanaka, "Proc. 5th Intern. Cong. Rheology,", Vol. 4, S. Onogi,
Ed., Tokyo Univ. Press., 1970, pp 251 and 259.

4. W. P. Mason, Trans. Amer. Soc. Mech. Engrs., 69, 359 (1947).

5. B. H. Zimm, J. Chem. Phys., 24, 269 (1956).

6. N. W. Tschoeg1, J. Chem. Phys., 39, 149 (1963); 40, 473 (1964).

7. J. L. Schrag and R. M. Johnson, Rev. Sci. Instr., in press.

8. M. H. Birnboim and L. J. Elyash, Bull. Amer. Phys. Soc., Ser. II, 11, 165 (1966).

9. Reference 2, pp 212, 214.

10. T. Ogasa and S. Imai, J. Chem. Phys., submitted, and private communication.

11. G. C. Berry, J. Chem. Phys., 44, 4553 (1966).

12. S. Kusamizu, L. A. Holmes, R. S. Moore, and J. D. Ferry, Trans. Soc. Rheology, 12, 562 (1968).

13. D. J. Massa and J. L. Schrag, in preparation.

14. W. H. Stockmayer and M. Kurata, Adv. Polym. Sci., 3, 196 (1963).

15. R. M. Johnson, Ph. D. Thesis, University of Wisconsin, 1970.

16. C. W. Pyun and M. Fixman, J. Chem. Phys. 42, 3838 (1965); 44, 2107 (1966).

17. A. S. Lodge and Y.-J. Wu, in preparation.

18. S. E. Lovell and J. D. Ferry, J. Phys. Chem., 65, 2274 (1961). 